\title{
Heavy quark supermultiplet excitations
}

\author{
R. Delbourgo and Dongsheng Liu \\ Physics Department, University of Tasmania, Hobart, Australia 7005
}

(9 December 1993)

\begin{abstract}
Lorentz-covariant wave functions for meson and baryon supermultiplets are simply derived by boosting $\mathrm{SU}(2)_{\text {spin }}$ representations corresponding to multiquark systems at rest.

11.30-j,11.30.Cp,11.30.Ly,12.50.Ch
\end{abstract}

Typeset using REVTEX 


\section{INTRODUCTION}

A number of articles have recently appeared [1] where descriptions of hadronic excitations containing one or more heavy quarks have been presented. All of these recent constructions have regarded the external velocity of the hadron $v$ as a four-vector parameter and have written the wave functions in terms of $v$ and of internal velocities $u$; in these schemes one encounters projections parallel and perpendicular to $v$ and tensors/spinors formed out of $v$, the several $u$ and the gamma matrices. Some of the descriptions are quite elaborate and involve complicated algebraic manoeuvres over Lorentz covariant objects before the final forms are attained. Occasionally the derivations are given in the Bethe-Salpeter framework [2] or variants thereof.

Most of these authors were (understandably) unaware that this problem was tackled many years ago [3] in the context of Reggeization of supermultiplet theory. At that time there was interest in constructing supermultiplet wavefunctions at arbitrary integer total angular momentum $J$ (or its Casimir generalization for the chosen supersymmetry group), before continuing somehow to complex $J$-values. Multispinor wavefunctions appearing in section IV of reference 3 were quoted abruptly without much elaboration and the formulae may therefore look rather mysterious today. Because this subject of hadronic excitations has come back into vogue in the context of heavy quark physics, we shall explain here the derivation of multiquark excitation functions and will take the opportunity to correct a few normalization factors in reference 3 . Then we shall go on to discuss the application to heavy quark composites.

The basic idea is extremely simple and direct: since all such supermultiplets are massive we can always proceed to the rest frame, where the four-velocity $v$ is a unit timelike vector pointing along the time axis. In that frame the only surviving space-time symmetry is the little group of the full Lorentz group associated with $v$, namely spatial $\mathrm{SU}(2)$, and the states fall into irreducible representations of it. We now know (as was not fully appreciated in the pre-bc year 1969) that hadrons are white composites of quarks and gluons; the standard 
picture views the mesons as made of one quark and an antiquark, while baryons are made of three quarks, plus a colourless mixture of any number of gluons and quark-antiquark pairs. Some models sometimes replace two quasi-free quarks by a single composite diquark, but the basic idea is essentially the same. The internal degrees of freedom and the binding mechanism - which is not completely understood at low energies - produce a bound multiquark state that carries appropriate quantum numbers; these can be nothing more than the $\mathrm{SU}(2)_{J}$ labels plus a possible multiplicity label $N$ for states which are repeated, as well as the internal flavour group quantum numbers which we have not bothered to expose. In other words the integrations over the internal momenta eventually lead, in the rest frame, to meson and baryon states

$$
\left[\phi_{a}^{\bar{b}}\right]_{\left\{m_{1} . . m_{L}\right\}}^{N} \text { and }\left[\psi_{a b c}\right]_{\left\{m_{1} . . m_{L}\right\}}^{N}
$$

where $m$ is a $\mathrm{O}(3)$ vector index corresponding to orbital excitation $L$, and $a, b, c$ stand for two-component spinor indices (barred for antiquarks). $\{.$.$\} represents a symmetrised tensor$

product and we have assumed above that all Kronecker traces over the $m$ indices are zero to make the orbital state irreducible with respect to $\mathrm{O}(3)$. In the next section we shall reduce these states with respect to total angular momentum $J$ and in the following section we will boost up the results to arbitrary velocity $v$. Finally we shall discuss the connection with Lagrangians, Bethe-Salpeter wavefunctions and other work.

\section{NON-RELATIVISTIC REDUCTION INTO $J$ REPRESENTATIONS}

The first task is to simplify the spin structure. Because the quark and antiquarks can in principle be acted upon by different spin groups (see section IV), we first reduce the multispinors into total spin states, disregarding their orbital quantum numbers,

$$
\begin{gathered}
\phi_{a}^{\bar{b}}=\left[\phi_{5} \delta_{a}^{\bar{b}}+\left(\sigma_{m}\right)_{a}^{\bar{b}} \phi_{m}\right] / \sqrt{2} \\
\psi_{a b c}=\psi_{\{a b c\}}+\psi_{\{a b\} c}+\psi_{[a b] c}=\left(\sigma_{2} \sigma_{m}\right)_{\{a b\}} \psi_{m c} / \sqrt{2}+\left(\epsilon_{a c} \psi_{b}+\epsilon_{b c} \psi_{a}\right) / \sqrt{6}+\epsilon_{a b} \psi_{c}^{\prime} / \sqrt{2} .
\end{gathered}
$$


Since antiparticles have opposite parity to particles we recognise the pseudoscalar state $\phi_{5}\left(0^{--}\right)$, the vector state $\vec{\phi}\left(1^{--}\right)$, the spin $1 / 2$ states $\psi, \psi^{\prime}$ and the spin $3 / 2$ state $\vec{\psi}_{c}$, obeying the irreducibility condition, $\sigma_{m} \psi_{m}=0$ which ensures that $\psi_{\{a b c\}}$ is symmetric. Note the occurrence of the $\sigma_{2}$ matrix which is the lowering operator for $\mathrm{SU}(2)$ and the charge conjugation matrix non-relativistically.

The next step is to combine spin and orbital factors into representations of total spin $J$. As far as the pseudoscalar meson excitations are concerned, there is nothing more to be done: $\phi_{5\left\{m_{1} . . m_{L}\right\}}$ stands for a state with parity $(-1)^{L+1}$ and $C P=1$; the vector meson excitations $(1 \times L)$ require reduction to states with $J=L+1, L, L-1$ as given below:

$$
\begin{aligned}
\phi_{m\left\{m_{1} \ldots m_{L}\right\}} & =\phi_{\left\{m m_{1} \cdots m_{L}\right\}}^{(L+1)}+\frac{1}{\sqrt{2} L} \sum_{k} i \epsilon_{m m_{k} n} \phi_{\left\{m_{1} \cdots \bar{k} \cdots m_{L} n\right\}}^{(L)} \\
& +\frac{1}{L} \sqrt{\frac{2 L-1}{2 L+1}} \sum_{k}\left[\delta_{m m_{k}} \phi_{\left\{m_{1} \cdots \bar{k} \cdots m_{L}\right\}}^{(L-1)}-\frac{2}{2 L-1} \sum_{l} \delta_{m_{k} m_{l}} \phi_{\left\{m m_{1} \cdots \bar{k} \bar{l} \cdots m_{L}\right\}}^{(L-1)}\right] ;
\end{aligned}
$$

a bar over an $\mathrm{O}(3)$ index, like $\bar{k}$, signifies that $m_{k}$ is missing from the tensor.

Turning to the baryons, one either needs to combine the orbital momentum with spin $1 / 2$ or with spin $3 / 2$. In the former case one arrives at states with $J=L+1 / 2$ and $J=L-1 / 2$, encapsulated by the decomposition

$$
\psi_{\left\{m_{1} . . m_{L}\right\}}=\psi_{\left\{m_{1} \cdots m_{L}\right\}}^{(L+1 / 2)}+\frac{1}{\sqrt{L(2 L+1)}} \sum_{k} \sigma_{m_{k}} \psi_{\left\{m_{1} \cdots \bar{k} \cdots m_{L}\right\}}^{(L-1 / 2)}
$$

while in the latter case one must reduce to $J=L+3 / 2, L+1 / 2, L-1 / 2$ and $L-3 / 2$ representations:

$$
\begin{gathered}
\psi_{m\left\{m_{1} \ldots m_{L}\right\}}=\psi_{\left\{m_{1} \cdots m_{L}\right\}}^{(L+3 / 2)}+ \\
\sqrt{\frac{3}{4 L(2 L+3)}\left[\sum_{k}\left(i \epsilon_{m m_{k} n} \psi_{\left\{m_{1} \cdots \bar{k} \cdots m_{L} n\right\}}^{(L+1 / 2)}+\sigma_{m_{k}} \psi_{\left\{m m_{1} \cdots \bar{k} \cdots m_{L}\right\}}^{(L+1 / 2)}\right)-\frac{L}{3} \sigma_{m} \psi_{\left\{m_{1} \cdots m_{L}\right\}}^{(L+1 / 2)}\right]+} \\
\sqrt{\frac{3(2 L-1)}{L(L+1)(2 L+1)} \sum_{k}\left[\left(\frac{2 \delta_{m m_{k}}}{3}-\frac{i \epsilon_{m m_{k} n} \sigma_{n}}{3}\right) \psi_{\left\{m_{1} \cdots \bar{k} \cdots m_{L}\right\}}^{(L-1 / 2)}-\sum_{l} \frac{2 \delta_{m_{k} m_{l}}}{2 L-1} \psi_{\left\{m m_{1} \cdots \bar{k} \bar{l} \cdots m_{L}\right\}}^{(L-1 / 2)}\right]+} \\
\frac{1}{L \sqrt{(L-1)(2 L+1)}} \sum_{k, l}\left[\left(\delta_{m m_{k}} \sigma_{m_{l}}-\frac{\left.2 \sigma_{m} \delta_{m_{k} m_{l}}\right)}{2 L-1}\right) \psi_{\left\{m_{1} \cdots \bar{k} \bar{l} \cdots m_{L}\right\}}^{(L-3 / 2)}-\sum_{n} \frac{2 \delta_{m_{k} m_{l}} \sigma_{m_{n}}}{2 L-1} \psi_{\left\{m m_{1} \cdots \bar{k} \bar{n} \cdots m_{L}\right\}}^{(L-3 / 2)}\right]
\end{gathered}
$$


These expressions are correctly normalized, in as much as $1=\sum_{J}\left|\psi_{\left\{m_{1} \cdots\right\}}^{(J)}\right|^{2}$, like the lefthand sides of Eqs. (2) to (4).

\section{BOOSTED WAVEFUNCTIONS}

It is not widely appreciated that Lorentz covariant expressions for particle wavefunctions are readily obtained by boosting the non-relativistic formulae. An incoming meson which is a composite of an incoming quark and antiquark must contain the projection factors $\left[\left(1+\gamma_{0}\right) / 2\right] \Gamma\left[\left(1-\gamma_{0}\right) / 2\right]$ in the rest frame in order to pick out the upper two components of the quark and the lower two components of the other quark. Likewise the non-relativistic expressions $\left(\sigma_{2}\right)_{[a b]}$ and $\left(\sigma_{2} \sigma_{m}\right)_{\{a b\}}$ should be interpreted as the upper $2 \times 2$ components of the four-component multispinors $\left[\left(1+\gamma_{0}\right) \gamma_{5} C / 2\right]_{\alpha \beta}$ and $\left[\left(1+\gamma_{0}\right) \gamma_{m} C / 2\right]_{\alpha \beta}$. From this point of view it is easy to understand why the properly boosted versions (any direction of $v$ ) of the wavefunctions (2) are

$$
[\phi(v)]_{\alpha}^{\beta}=\left[(1+\gamma \cdot v)\left(\gamma_{5} \phi_{5}(v)-\gamma^{\mu} \phi_{\mu}(v)\right) / 2 \sqrt{2}\right]_{\alpha}^{\beta},
$$

where vector indices on fields are orthogonal to $v$, or $v^{\mu} \phi_{\mu}=0$, signifying that the $\phi$ are polarization vectors. Also the generalization of the non-relativistic condition on the vector-spinor, $\vec{\sigma} \cdot \vec{\psi}=0$ reads $\gamma \cdot \psi=v \cdot \psi=0$. Results of this type were originally derived $\left[\begin{array}{ll}\text { by carrying } \\ \text { b }\end{array}\right.$ out Lorentz-covariant reductions of multispinors, using Bargmann-Wigner equations acting on each Dirac spinor index and solving the constraint and symmetry conditions. (They have been rediscovered several times in different ways.) In the light of experience this is an unnecessarily complicated way of proceeding: one simply "solves" the equations [5] in the rest frames, a totally trivial step, and boosts up to arbitrary $v$, as above. The only other substitutions we must be careful with are for spin matrices and the Kronecker delta,

$$
\sigma_{m} \rightarrow v^{\nu} \sigma_{\nu \mu} \gamma_{5} \equiv w_{\mu}, \quad \delta_{m n} \rightarrow-\eta_{\mu \nu}+v_{\mu} v_{\nu} \equiv d_{\mu \nu}(v)
$$

where we have assumed that $v$ has unit length on-shell. 
With this point made, we may readily understand why the relativistic versions of Eqs. (2), (3) and (4) are

$$
\begin{aligned}
& \phi_{\mu\left\{\mu_{1} . . \mu_{L}\right\}}=\phi_{\left\{\mu \mu_{1} \cdots \mu_{L}\right\}}^{(L+1)}+\frac{1}{\sqrt{2} L} \sum_{k} i v^{\lambda} \epsilon_{\lambda \mu \mu_{k} \nu} d^{\nu \nu^{\prime}} \phi_{\left\{\mu_{1} \cdots \bar{k} \cdots \mu_{L} \nu^{\prime}\right\}}^{(L)} \\
& +\frac{1}{L} \sqrt{\frac{2 L-1}{2 L+1}} \sum_{k}\left[d_{\mu \mu_{k}} \phi_{\left\{\mu_{1} \cdots \bar{k} \cdots \mu_{L}\right\}}^{(L-1)}-\frac{2}{2 L-1} \sum_{l} d_{\mu_{k} \mu_{l}} \phi_{\left\{\mu \mu_{1} \cdots \bar{k} \bar{l} \cdots \mu_{L}\right\}}^{(L-1)}\right] \text {; } \\
& \psi_{\left\{\mu_{1} \ldots \mu_{L}\right\}}=\psi_{\left\{\mu_{1} \cdots \mu_{L}\right\}}^{(L+1 / 2)}+\frac{1}{\sqrt{L(2 L+1)}} \sum_{k} w_{\mu_{k}} \psi_{\left\{\mu_{1} \cdots \bar{k} \cdots \mu_{L}\right\}}^{(L-1 / 2)} \\
& \psi_{\mu\left\{\mu_{1} \ldots \mu_{L}\right\}}=\psi_{\left\{\mu \mu_{1} \cdots \mu_{L}\right\}}^{(L+3 / 2)}+
\end{aligned}
$$

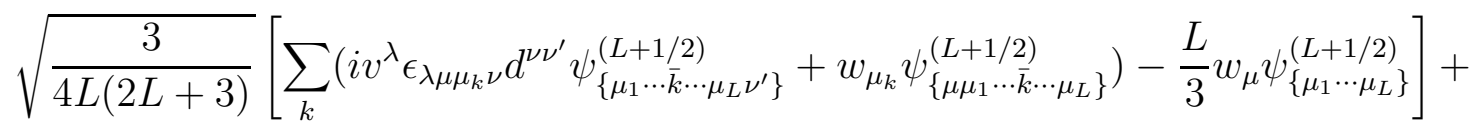

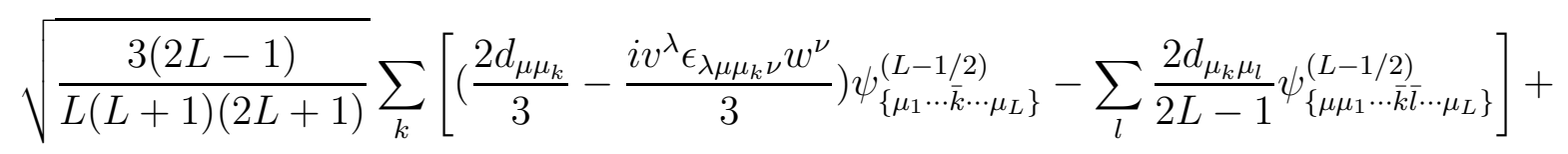

$$
\begin{aligned}
& \frac{1}{L \sqrt{(L-1)(2 L+1)}} \sum_{k, l}\left[\left(d_{\mu \mu_{k}} w_{\mu_{l}}-\frac{2 w_{\mu} d_{\mu_{k} \mu_{l}}}{2 L-1}\right) \psi_{\left\{\mu_{1} \cdots \bar{k} \bar{l} \cdots \mu_{L}\right\}}^{(L-3 / 2)}-\sum_{n} \frac{2 d_{\mu_{k} \mu_{l}} w_{\mu_{n}}}{2 L-1} \psi_{\left\{\mu \mu_{1} \cdots \bar{k} \bar{l} \bar{n} \cdots \mu_{L}\right\}}^{(L-3 / 2)}\right] \text {. }
\end{aligned}
$$

These expressions make no reference to the internal momenta, nor should they. The final covariant wavefunctions carry the quantum numbers associated with external momentum and its little group, no more and no less. For instance, the first excited states $(L=1)$ of mesons $\left(0^{++}, 1^{-+}, 2^{++}\right)$and baryons $\left(1 / 2^{-}, 3 / 2^{-}, 5 / 2^{-}\right)$are represented by

$$
\begin{gathered}
\phi_{\mu \nu}=\phi_{\{\mu \nu\}}^{(2)}-\frac{i}{\sqrt{8}} v^{\lambda} \epsilon_{\lambda \mu \nu \kappa} \eta^{\kappa \kappa^{\prime}} \phi_{\kappa^{\prime}}^{(1)}+\frac{1}{\sqrt{3}} d_{\mu \nu} \phi^{(0)} ; \quad \psi_{\mu}=\psi_{\mu}^{(3 / 2)}+w_{\mu} \psi^{(1 / 2)} / \sqrt{3} \\
\psi_{\mu \nu}=\psi_{\{\mu \nu\}}^{(5 / 2)}+\sqrt{\frac{3}{20}}\left[-i v^{\lambda} \epsilon_{\lambda \mu \nu \kappa} \eta^{\kappa \kappa^{\prime}} \psi_{\kappa^{\prime}}^{(3 / 2)}+w_{\nu} \psi_{\mu}^{(3 / 2)}-\frac{1}{3} w_{\mu} \psi_{\nu}^{(3 / 2)}\right]+\frac{1}{3 \sqrt{2}}\left[2 d_{\mu \nu}-i v^{\lambda} \epsilon_{\lambda \mu \nu \kappa} w^{\kappa}\right] \psi^{(1 / 2)}
\end{gathered}
$$

The only place where a connection may be made with the internal, relative momentaand the dynamics that leads to such bound states - is via the dependence of the masses on total angular momentum, $p^{2}=M_{J}^{2}(N)$, and on the (suppressed) quantum number $N$, differentiating between states of the same $J$. We shall turn to this aspect of the problem now. 


\section{CONNECTION WITH LAGRANGIANS AND BOUND STATE EQUATIONS}

It will pay us to re-examine the origin of supermultiplet symmetries as the older interpretation [4] differs slightly from the modern viewpoint advocated by the Mainz and Harvard schools [6]. Consider a set of quark fields with different masses described by the Lagrangian,

$$
\mathcal{L}=\sum_{F, C} \int d^{4} x \bar{Q}_{F C}(x)\left[\gamma \cdot(i \partial-g A(x))-m_{F}\right] Q_{F C}(x)+\mathcal{L}_{A}+\mathcal{L}_{\mathrm{ew}}
$$

where $F$ stands for the flavour and $C$ for colour and $A$ is the (matrix-valued) gluon octet field. If we disregard gluon interactions and make a Fourier transformation to momentum space we get

$$
\mathcal{L}_{\text {free }}=\sum_{F, C} \int d^{4} p \bar{q}_{F C}(p)\left[\gamma \cdot p-m_{F}\right] q_{F C}(p)
$$

The early relativistic interpretations of the Wigner supermultiplet symmetry for particle physics [0] neglected mass differences between quarks in order to show that the momentum space wavefunctions admitted an $S U\left(2 N_{F}\right) \times S U\left(2 N_{F}\right)$ invariance of $\mathcal{L}$. It was Isgur and Wise [8] who realised that this assumption of mass equality was not needed and who extended the concept to heavy quarks like $b$ and $c$. Heeding their lesson, let us change to velocity space by redefining $h_{F C}(v)=M^{5 / 2} q(p)$ as a new heavy quark field (normalised to 1 compared with $q(p)$ which was normalised to $\left.M^{-5 / 2}\right)$. The free part of the Lagrangian

$$
\mathcal{L}=\sum_{F, C} \int d^{4} v \bar{h}_{F C}(v)[\gamma \cdot v-1] h_{F C}(v)+\mathcal{L}_{\text {int }}(A)+\mathcal{L}_{\mathrm{ew}}
$$

then admits a $U\left(2 N_{F}\right) \times U\left(2 N_{F}\right)$ symmetry between the quark fields at fixed velocity. This is most readily seen in the rest frame $v_{0}=(1,0,0,0)$ with $h\left(v_{0}\right)$ obeying the projection equation $\left(\gamma_{0}-1\right) h\left(v_{0}\right)=0$. The little group generators then consist of

$$
\left[1, \gamma_{0}, \vec{\sigma}, \vec{\sigma} \gamma_{0}\right] \quad \times \quad T_{F}^{F^{\prime}}
$$

where $T$ are the generators of the $U\left(N_{F}\right)$ flavour algebra. When boosted to any velocity $v$,

$$
S\left(L_{v}\right) \gamma_{0} S^{-1}\left(L_{v}\right)=\gamma \cdot v
$$


the free spinors $h(v)=S\left(L_{v}\right) h\left(v_{0}\right)$ retain that supersymmetry but we should now interpret the generators as

$$
\left[1, v \cdot \gamma, w_{\mu}, w_{\mu} v \cdot \gamma\right] \times T_{F}^{F^{\prime}}
$$

Note that we have not toed the modern party line which considers the heavy quark field $h$ to be a full function of space-time $x$ with $v$ regarded as an external parameter (eventually identified with the velocity of the heavy hadron, one we may need to integrate over at the end of the day [9]). Instead we have surmised that $v$ is associated with the Fourier transform space, so that integration over $v$ is automatic and $x$ does not appear at all. The similarity transformation $S\left(L_{v}\right)$ which carries spinors from the rest frame $v_{0}$ to any $v$ can be construed as the Fourier transform of a coordinate-space Foldy-Wouthuysen-like transformation [10] applying to $h(x)$.

The usefulness of this concept hinges upon consideration of the quark-gluon interaction

$$
\mathcal{L}_{\text {int }}=\sum_{C, F} \int d^{4} v d^{4} v^{\prime} g \bar{h}(v) A\left(v-v^{\prime}\right) \cdot T h\left(v^{\prime}\right) / m_{F} .
$$

where the fluctuations over the gluon field $A$ produce a distribution over momenta of order $\Lambda_{\mathrm{QCD}}$. Since this will lead to corrections $\Lambda_{\mathrm{QCD}} / m_{F}$ to the free system, these will be most substantial for the lighter quarks and will become negligible as the quark mass $m_{F}$ gets ever larger, which Isgur and Wise [8] first observed. Thus one concludes that the really significant supersymmetry when QCD becomes operational is for the heavy flavours. However we believe that the formulation above, where the heavy quarks fields are functions of fourvelocity $v$ alone and not $x$ and $v$ simultaneously, brings this feature out much more clearly and elegantly. It may be possible to carry out a further transformation,

$$
Q(x) \rightarrow T\left[\exp i \int_{-\infty}^{x} A(\xi) \cdot d \xi\right] Q(x)
$$

in order to simplify the quark-gluon interaction (at the expense of the measure in the functional integral over the fields), but that is irrelevant for the present discussion.

To finish off let us briefly compare our formulation of hadronic excitations with the work of others [1], [2]. The early work by Isgur et al concerning strong and semileptonic 
decays of excited hadrons was cast in a non-covariant framework and based on an $S U\left(N_{F}\right)_{W}$ symmetry [11], associated with a three-system coupling, and used standard Clebsch-Gordan technology. It was shown to be entirely equivalent to the covariant approach by Hussain, Korner and Thompson [2]. In our language the interactions between an excited meson state ( $L$, incoming velocity $v$ ) and the two ground states $\left(L=0\right.$, outgoing velocities $\left.v_{1}, v_{2}\right)$ is written as

$$
G_{L} \operatorname{Tr}\left[\phi_{\left\{\mu_{1} \cdots \mu_{L}\right\}}(v)\left\{\phi\left(-v_{1}\right), \phi\left(-v_{2}\right)\right\}\right]\left(v_{1}-v_{2}\right)^{\mu_{1}} \cdots\left(v_{1}-v_{2}\right)^{\mu_{L}},
$$

and similarly for baryons; flavour quantum numbers are implied and traced over as well. In the rest frame of the decaying particle one may easily recover the expressions of Isgur et al, since the summation over the relative momentum indices produces the appropriate $S U\left(N_{F}\right)$ rotation function [3].

Falk and Luke [1] constructed states which are very similar to ours. However we differ from their approach in that we have recognised that the heavy quark must be accompanied by other quarks to produce the correct colourless state; after identifying them and their spin contributions (which are added to the heavy quark) we have afterwards appended the excitation numbers $(L, N)$ corresponding to the quark sea and gluons. The similarity of their spinor wavefunctions with ours can be established by noticing that the Pauli-Lubanski spin $w_{\mu}$ acting on a spinor $\psi(v)$ gives

$$
v^{\nu} \sigma_{\nu \mu} \gamma_{5} \psi(v) \rightarrow i\left(\gamma_{\mu}+v_{\mu}\right) \gamma_{5} \psi(v)
$$

The connection with Bethe-Salpeter $\Phi$ wavefunctions [2] is more distant. In this paper we have adopted the attitude that the excited states are projected out from the wave-functions using an orthogonal set of functions of the relative velocities $u$; specifically 12 for the mesons say,

$$
\phi(v)_{L M}^{N}=\int \Phi(v, u) \mathcal{Y}_{L M}^{N}(u) d^{4} u
$$

with $v^{2}=1$ or $p^{2}=m_{N L}^{2}$ since we are at the meson pole. All we can be sure of is that the orthogonal functions $\mathcal{Y}$ contain the spherical harmonic $Y_{L M}(\mathbf{u})$ in the rest frame $v_{0}$ of the 
meson. Naturally, the dynamics which comes via the Bethe-Salpeter kernel (and is presumably dominated by non-perturbative gluon exchange) will dictate the remaining dependence of $\mathcal{Y}^{N}$ on $u . v$ and $u^{2}$, viz. some linearly independent combinations of hyperspherical harmonics. An alternative approach [2] is the leave the $\Phi$ of initial and final hadrons intact and carry out an internal integration over projections involving relative velocities of the current matrix elements. The only point one can be reasonably sure of in both approaches is that the degree to which the quarks in the meson are off-shell (or spread in $|u|$ ) is of order $\Lambda_{\mathrm{QCD}} / m_{F}$. It could be that a Bethe-Salpeter equation, something like

$$
\left[\gamma \cdot\left(\mu_{1} p+k\right)-m_{1}\right] \Phi(p, k)\left[\gamma \cdot\left(\mu_{2} p-k\right)+m_{2}\right]=\int \gamma^{\nu} \lambda^{c} . \Phi\left(p, k^{\prime}\right) \cdot \gamma_{\nu} \lambda^{c} D\left(k-k^{\prime}\right) d^{4} k^{\prime}
$$

will do the trick, where the exchange propagator $D\left(k-k^{\prime}\right)$ is as singular as $\Lambda^{2} /\left(k-k^{\prime}\right)^{4}$ at small momentum transfer. Gudehus [2] indicates that the details may not be very important anyway in obtaining the requisite heavy quark symmetry and wavefunctions, and in deriving the supermultiplet universal form factors, at least for the lowest state $N=0$. This is a topic that could bear closer investigation.

\section{ACKNOWLEDGMENTS}

We acknowledge support from the Australian Research Council under grant number A69231484 and thank Dr. Kreimer for commenting on our work. 


\section{REFERENCES}

* EMail: Bob.Delbourgo@Phys.UTas.Edu.Au and Liu@PhysVax.Phys.UTas.Edu.Au

[1] N. Isgur and M. Wise, Phys. Rev. Lett. 66, 1130 (1991) and Phys. Rev. D43, 819 (1991); N. Isgur, M. B. Wise and M. Youssefmir, Phys. Lett. 254, 215 (1991); A. Falk and M. Luke, Phys. Lett. 292, 119 (1992); A. Falk, Nucl. Phys. B378, 79 (1992); S. Balk, F. Hussain, J. G. Korner and G. Thompson, Z. Phys. C59, 283 (1993); F. Hussain, G. Thompson and J. G. Korner, Mainz preprint MZ-TH/93-23 (1993).

[2] T. Gudehus, Phys. Rev. 184, 1788 (1969); F. Hussain, J. G. Korner and G. Thompson, Ann. Phys. 206334 (1991); Y-B Dai, G-S Huang and H-Y Jin, Z. Phys. C60, 527 (1993).

[3] R. Delbourgo and A. Salam, Phys. Rev. 186, 1516 (1969) and Phys. Lett. 28B, 497 (1969).

[4] A. Salam, R. Delbourgo and J. Strathdee, Proc. Roy. Soc. 284A, 146 (1965) and ibid Proc. Roy. Soc. 285A, 312 (1965); K. Bardakci, J. M. Cornwall, P. G. O. Freund and B. W. Lee, Phys. Rev. Lett. 14, 264 (1965); M. A. Beg and A. Pais, Phys. Rev. Lett. 14, 267 (1965); B. Sakita and K. C. Wali, Phys. Rev. 139, B1355 (1965).

[5] R. Delbourgo, M.A. Rashid, A. Salam and J. Strathdee, The U(12) Symmetry (IAEA, Vienna, 1965).

[6] E. Eichten and B. Hill, Phys. Lett. B234, 511 (1990); A. F. Falk, H. Georgi, B. Grinstein and M. B. Wise, Nucl. Phys. B343, 1 (1990); T. Mannel, W. Roberts and Z. Ryzak, Nucl. Phys. B368, 204 (1992); J. G. Korner and G. Thompson, Phys. Lett. B264, 185 (1991).

[7] F. Gursey and L. Radicati, Phys. Rev. Lett. 299, 13 (1964); B. Sakita, Phys. Rev. 136, B1756 (1964); A. Pais, Phys. Rev. Lett. 13, 175 (1964).

[8] N. Isgur and M. Wise, Phys. Lett. B232, 113 (1989); ibid B237, 527 (1990). 
[9] H. Georgi, Phys. Lett. 240, 447 (1990) and Nucl. Phys. 348, 293 (1991)

[10] L. L. Foldy and S. A. Wouthuysen, Phys. Rev. 78, 29 (1950); S. Tani, Prog. Theor. Phys. 6, 267 (1951); H. J. Melosh, Phys. Rev. D9, 1095 (1974); J. G. Korner and G. Thompson, Phys. Lett. B264, 185 (1991); A. Das, University of Rochester preprint, UR-1325 (1993).

[11] H. J. Lipkin and S. Meshkov, Phys. Rev. Lett. 14, 670 (1965); K. J. Barnes, ibid 14, 798 (1965); D. Horn, H. J. Lipkin and S. Meshkov, Phys. Rev. Lett. 17, 1200 (1966).

[12] G. C. Wick, Phys. Rev. 96, 1124 (1954); R. E. Cutkosky, ibid 1135 (1954); W. Kummer, Nuovo Cim. 31, 219 (1964); S. Mandelstam, Proc. Roy. Soc. 237A, 496 (1966); R. Delbourgo, A. Salam and J. Strathdee, Phys. Lett. 21, 455 (1966) and Nuovo Cim. 50, $193(1967)$. 